\title{
The obesity paradox in pulmonary arterial hypertension: the Scottish perspective
}

\section{To the Editor:}

Pulmonary arterial hypertension $(\mathrm{PAH})$ is a rare disease characterised by progressive pulmonary vascular remodelling, resulting in right ventricular failure, which is the principal cause of death [1]. Excessive bodyweight is a well-established independent risk factor for the development of cardiovascular disease and mortality [2]. However, data have emerged for a variety of chronic cardiovascular diseases, particularly heart failure, showing lower long-term mortality in obese patients [3]. This has been termed the "obesity paradox". It challenges clinical reasoning and the significant healthcare expenditure directed toward weight loss in the general population.

An analysis of the US-based REVEAL (Registry to Evaluate Early and Long-term PAH Disease Management) found obese PAH patients had a reduced risk of death, despite important limitations of this registry [4]; foremost, the inclusion of prevalent patients and disparities in the standard of care available. Additional studies of pulmonary hypertension $(\mathrm{PH})$ have shown obesity carries a survival benefit across a number of patient populations including incident patients [5-10].

Explanations for this paradox include obese patients having greater metabolic reserve to meet the increased catabolic burden, oxidative stress and systemic inflammation associated with heart failure [11]. However, this is a contentious area and many studies supporting the hypothesis are only observational. They may miss the effect of illness-induced weight-loss. Obese patients may also present earlier and be treated more appropriately earlier in time [12]. Indeed, a large recent study conducted in the French reference centre for $\mathrm{PH}$ has suggested there is no protective effect from obesity [13]. However, this population included a significant proportion of drug-induced PAH patients, which may have skewed the results.

We investigated the Scottish Pulmonary Vascular Unit (SPVU) incident PAH population in order to evaluate whether obesity carries a survival benefit. All patients with suspected PAH in Scotland (population 5.1 million) are referred to the SPVU prior to right heart catheterisation, so this study represents a true national incident population.

We performed a retrospective longitudinal observational study of all consecutive, newly diagnosed idiopathic and hereditary PAH patients managed by the SPVU between 1997 and 2016. Parameters collected at diagnosis included haemodynamics (right atrial pressure (RAP), pulmonary artery pressure (PAP) and pulmonary capillary wedge pressure (PCWP)), N-terminal pro-brain natriuretic peptide (NT-proBNP), 6-min walk distance (6MWD), functional class (FC), age and body mass index (BMI). All patients were treatment-naive prior to right heart catheterisation. The treatment commenced was at the discretion of the treating specialist, in accordance with contemporary guidelines. World Health Organization-defined BMI categories were used, with a BMI of $30 \mathrm{~kg} \cdot \mathrm{m}^{-2}$ representing obesity. A survival analysis including Kaplan-Meier curves and Cox regression analysis was performed.

$@$ ERSpublications

Analysis of pulmonary arterial hypertension patients in Scotland across 20 years supports a recent French study suggesting there is no protective effect from obesity for this disease, in contrast to emerging evidence from the USA and China http://bit.ly/34WCZ7W

Cite this article as: McLean LL, Pellino K, Brewis M, et al. The obesity paradox in pulmonary arterial hypertension: the Scottish perspective. ERJ Open Res 2019; 5: 00241-2019 [https://doi.org/ 10.1183/23120541.00241-2019]. 
The analysis included 266 patients, 260 with idiopathic PAH and six with hereditary PAH. The prevalence of obesity was $35.7 \%$. The nonobese group (BMI $<30 \mathrm{~kg} \cdot \mathrm{m}^{-2}$ ) comprised 171 patients, with a mean BMI of $24.7 \pm 3.3 \mathrm{~kg} \cdot \mathrm{m}^{-2}$. The obese group (BMI $\geqslant 30 \mathrm{~kg} \cdot \mathrm{m}^{-2}$ ) comprised 95 patients, with a mean BMI of $35.9 \pm 5.7 \mathrm{~kg} \cdot \mathrm{m}^{-2}$. Mean age at diagnosis was $60.9 \pm 18.2$ years and $59.18 \pm 14.3$ years for the nonobese and obese groups, respectively. $49 \%$ of the nonobese group were female, compared to $77 \%$ in the obese group $(\mathrm{p}<0.001)$. Cardiac index, pulmonary vascular resistance, NT-proBNP and 6MWD were similar for both groups. The percentage of patients in FC I or II was higher in the nonobese group, $18.7 \%$ versus $14.9 \%$ for the obese group, although this did not reach statistical significance $(\mathrm{p}=0.054)$.

A number of haemodynamic parameters were significantly worse in the obese group. Mean RAP was $9.8 \pm 5.5 \mathrm{mmHg}$ for the obese group and $7.5 \pm 5.1 \mathrm{mmHg}$ for the nonobese group $(\mathrm{p}<0.001)$. Mean PAP was $51.7 \pm 10.7 \mathrm{mmHg}$ for the obese group and $48.3 \pm 13.5 \mathrm{mmHg}$ for the nonobese group ( $\mathrm{p}=0.001)$. Mean PCWP was $8.6 \pm 3.9 \mathrm{mmHg}$ for the obese group and 7.5 $\pm 3.1 \mathrm{mmHg}$ for the nonobese group $(\mathrm{p}=0.029)$.

Using Kaplan-Meier survival analysis (figure 1), we found no significant difference in survival between the nonobese and obese groups $(\mathrm{p}=0.358)$. Cox regression survival analysis using a multivariable model found neither BMI nor obesity were significant prognostic indicators of survival, only age, NT-proBNP, 6MWD and female sex were. BMI significantly correlated only with RAP $(r=0.242, p<0.001)$ and PAP $(r=0.221$, $\mathrm{p}<0.001)$ using Spearman rank correlation.

Further variations to our model were made in order to test whether there was any obesity-mortality interaction. However, no significant difference in survival was observed when comparing overweight to nonoverweight individuals (BMI $\geqslant 25$ versus $<25 \mathrm{~kg} \cdot \mathrm{m}^{-2}$ ), excluding severely obese individuals $\left(\mathrm{BMI} \geqslant 40 \mathrm{~kg} \cdot \mathrm{m}^{-2}\right)$, or separating the cohorts based on age, using age separations at 50 and 65 years.

We acknowledge there are limitations in this retrospective, single-centre observational study. Recruitment of patients over a 20-year period may ignore significant changes in the demographics and treatment of $\mathrm{PAH}$ patients. Comorbid conditions including diabetes were not available for analysis. BMI was also the only available measure of adiposity; this does not account for adipose distribution and the effects of oedema at the time of diagnosis. BMI measurements were only recorded once at baseline but decreases in BMI over time may have occurred. The results may not be generalisable to patients with other group 1 $\mathrm{PAH}$ and $\mathrm{PH}$ aetiologies, and further studies are needed here. The strengths of this study are that the cohort is a large population of incident, treatment-naïve idiopathic and hereditary PAH patients only, assessed by specialists in a multidisciplinary team, with a standardised, high quality of treatment available as part of the UK's National Health Service.

In contrast to this study, some earlier studies supporting the obesity hypothesis have focused on prevalent patients [4] and patients with several PAH [8,9] or PH [7] aetiologies. Agarwal et al. [10] studied 18450 US-based in-hospital PAH patients and found obese patients had lower adjusted odds of mortality. However, this relied on a database using diagnostic codes rather than haemodynamics, with limited BMI information. The access to healthcare and specialised treatment may also be variable in some US studies [4]. A Chinese study of incident, treatment-naïve idiopathic PAH patients observed the obesity paradox

FIGURE 1 Kaplan-Meier survival analysis comparing the obese and nonobese groups. $\mathrm{p}=0.358 \quad$ log rank).

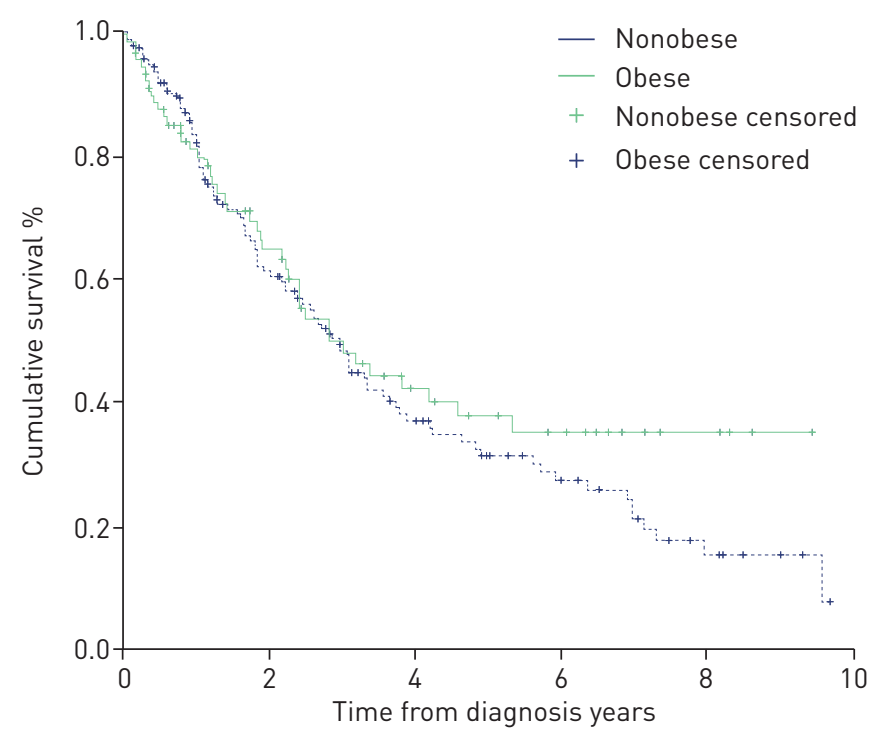


was present, but included only three obese patients and had very limited availability to PAH-targeted treatments, due to cost [5].

Moreover, there is no established mechanism for this obesity effect. Some authors have recognised the possibility of it being a statistical artefact or confounding factors being responsible [4, 14]. Indeed, WEATHERALD et al. [13] recently reported no overall association of obesity or BMI with mortality amongst a population of 1255 idiopathic, heritable and drug-induced PAH patients from the French Pulmonary Hypertension Network registry.

Our present study supports the conclusion of this larger, multicentre French study. This holds true despite important differences between the cohorts of both studies. The French study included many patients with drug- and toxin-induced PAH, representing as much as $29 \%$ of the obese group. Rates of obesity were lower in the French cohort ( $30 \%$ versus $36 \%$ with BMI $\geqslant 30 \mathrm{~kg} \cdot \mathrm{m}^{-2}$ ). Baseline parameters including FC, 6MWD and haemodynamics were worse in the SPVU cohort. However, both cohorts are similar in terms of age, BMI and gender splits. WEATHERALD et al. [13] also reported significantly worse haemodynamic parameters in their obese group compared to their nonobese group, as have we.

In conclusion, this study of idiopathic and heritable PAH patients demonstrates no survival benefit from obesity, and mortality was not significantly associated with BMI or obesity. We hope this gives further support to recent evidence from WeATHERALD et al. [13] suggesting the obesity paradox is not present in PAH.

Luke Lawson McLean ${ }^{1}$, Katherine Pellino ${ }^{2}$, Melanie Brewis ${ }^{2}$, Andrew Peacock ${ }^{2}$, Martin Johnson ${ }^{2}$ and Alistair Colin Church ${ }^{1,2}$

${ }^{1}$ College of Medical, Veterinary and Life Sciences, University of Glasgow, Glasgow, UK. ${ }^{2}$ Scottish Pulmonary Vascular Unit, Golden Jubilee National Hospital, Clydebank, UK.

Correspondence: Luke Lawson McLean, College of Medical, Veterinary and Life Sciences, Wolfson Medical School Building, University of Glasgow, University Avenue, Glasgow, G12 8QQ, UK. E-mail: 1.lawsonmclean@nhs.net

Received: 11 Sept 2019 | Accepted: 16 Sept 2019

Support statement: This study was supported by British Lung Foundation grant SS16/04. Funding information for this article has been deposited with the Crossref Funder Registry.

Conflict of interest: L.L. McLean reports that he received a Summer Studentship Award 2016 from the British Lung Foundation during the conduct of the study. K. Pellino has nothing to disclose. M. Brewis has nothing to disclose. A. Peacock has nothing to disclose. M. Johnson has nothing to disclose. A.C. Church has nothing to disclose.

\section{References}

1 Galie N, Humbert M, Vachiery JL, et al. 2015 ESC/ERS Guidelines for the diagnosis and treatment of pulmonary hypertension: The Joint Task Force for the Diagnosis and Treatment of Pulmonary Hypertension of the European Society of Cardiology (ESC) and the European Respiratory Society (ERS) Endorsed by: Association for European Paediatric and Congenital Cardiology (AEPC), International Society for Heart and Lung Transplantation (ISHLT). Eur Heart J 2016; 37: 67-119.

2 Van Gaal LF, Mertens IL, De Block CE. Mechanisms linking obesity with cardiovascular disease. Nature 2006 444: 875-880.

3 Flegal KM, Kit BK, Orpana H, et al. Association of all-cause mortality with overweight and obesity using standard body mass index categories: a systematic review and meta-analysis. JAMA 2013; 309: 71-82.

4 Poms AD, Turner M, Farber HW, et al. Comorbid conditions and outcomes in patients with pulmonary arterial hypertension. Chest 2013; 144: 169-176.

5 Hu EC, He JG, Liu ZH, et al. Survival advantages of excess body mass index in patients with idiopathic pulmonary arterial hypertension. Acta Cardiol 2014; 69: 673-678.

6 Zeng W-J, Sun Y-J, Gu Q, et al. The impact of pulmonary arterial hypertension-targeted therapy on survival in Chinese patients with idiopathic pulmonary arterial hypertension. Pulm Circ 2012; 2: 373-378.

7 Zafrir B, Adir Y, Shehedeh W, et al. The association between obesity, mortality and filling pressures in pulmonary hypertension patients; the "obesity paradox". Respir Med 2013; 107: 139-146.

8 Benza RL, Gomberg-Maitland M, Naeije R, et al. Prognostic factors associated with increased survival in patients with pulmonary arterial hypertension treated with subcutaneous treprostinil in randomized, placebo-controlled trials. J Heart Lung Transplant 2011; 30: 982-989.

9 Mazimba S, Holland E, Nagarajan V, et al. Obesity paradox in group 1 pulmonary hypertension: analysis of the NIH Pulmonary Hypertension registry. Int J Obes (Lond) 2017; 41: 1164-1168.

10 Agarwal M, Agrawal S, Garg L, et al. Relation between obesity and survival in patients hospitalized for pulmonary arterial hypertension (from a nationwide inpatient sample database 2003 to 2011). Am J Cardiol 2017; 120: 489-493.

11 Arena R, Lavie CJ. The obesity paradox and outcome in heart failure: is excess bodyweight truly protective? Future Cardiol 2010; 6: 1-6. 
12 Lavie CJ, Sharma A, Alpert MA, et al. Update on obesity and obesity paradox in heart failure. Prog Cardiovasc Dis 2016; 58: 393-400

13 Weatherald J, Huertas A, Boucly A, et al. Association between BMI and obesity with survival in pulmonary arterial hypertension. Chest 2018; 154: 872-881.

14 Lawlor DA, Hart CL, Hole DJ, et al. Reverse causality and confounding and the associations of overweight and obesity with mortality. Obesity 2006; 14: 2294-2304. 